\title{
On Stability of Fixed Points for Multi-Valued Mappings with an Application
}

\author{
Qi-Qing Song \\ College of Science, Guilin University of Technology, Guilin 541004, China \\ Correspondence should be addressed to Qi-Qing Song; songqiqing@gmail.com
}

Received 12 November 2013; Accepted 22 January 2014; Published 27 February 2014

Academic Editor: Salvador Romaguera

Copyright (C) 2014 Qi-Qing Song. This is an open access article distributed under the Creative Commons Attribution License, which permits unrestricted use, distribution, and reproduction in any medium, provided the original work is properly cited.

\begin{abstract}
This paper studies the stability of fixed points for multi-valued mappings in relation to selections. For multi-valued mappings admitting Michael selections, some examples are given to show that the fixed point mapping of these mappings are neither upper semi-continuous nor almost lower semi-continuous. Though the set of fixed points may be not compact for multi-valued mappings admitting Lipschitz selections, by finding sub-mappings of such mappings, the existence of minimal essential sets of fixed points is proved, and we show that there exists at least an essentially stable fixed point for almost all these mappings. As an application, we deduce an essentially stable result for differential inclusion problems.
\end{abstract}

\section{Introduction}

In [1], the conception of an essential fixed point was introduced by Fort for continuous functions on a compact subset of a metric space (Brouwer type fixed points); the idea was applied to analyze many stabilities of solutions for many kinds of nonlinear problems, such as vector quasi-equilibrium problems, coincident points, and Nash equilibrium problems (see [2-4]).

In fact, the stability of fixed points is connected with continuities of fixed points mappings. In [5], some generic continuities of semicontinuous functions were obtained, which have also been used to analyze many problems (see [6-9]). In hyperconvex metric spaces, the generic stability of fixed points for upper semicontinuous mappings (Fan-Glicksberg type fixed points) was obtained (see [10]).

Generally, the existence of essential Brouwer type fixed points cannot be guaranteed, which was used to prompt the study of essential components of these fixed points in [11], and the existence of such essential components was proved. Further, essential sets of Brouwer type fixed points were introduced (see [12]). For upper semicontinuous multivalued mappings, the existence of essential connected components of fixed points (Kakutani type fixed points) was also obtained in [13].
These sets of many kinds of solutions abovementioned and others (e.g., [14]) are compact, which facilitates the analysis of generic stabilties, essential sets, and essential components. It is well known that every lower semicontinuous function (Michael type function) from a paracompact space to the nonempty, closed, and convex subsets of a Banach space admits a continuous selection (see [15, Theorem 1] or [16, Theorem 3.2]). Noting the Brouwer's fixed point theorem, the result for Michael's continuous selection implies that each lower semi-continuous self mapping with nonempty closed convex values on a compact convex set of a normed linear space has a fixed point (Michael type fixed point). However, in this paper, some examples show that the set of Michael type fixed points need not be compact.

The aim of this paper is to study the stability of multivalued mappings concerning selections. Firstly, the multivalued mappings admitting a continuous selection (which includes Mychael type functions) are considered. The fixed point set of these mappings is not necessarily compact. The fixed point mapping for these mappings is neither upper semicontinuous nor almost lower semicontinuous. Secondly, the multivalued mappings admitting Lipschitz selections are studied. The fixed point set of these mappings need not also be compact. By finding an upper continuous submapping with compact values of each such mapping, the existence of minimal 
essential sets of fixed points is proved, and we show that there exists at least one essentially stable fixed point for most of these mappings. Finally, we deduce a stability result for differential inclusion problems as an application.

\section{Preliminaries and Motivations}

Let $E$ and $Y$ be two metric spaces. Firstly, we recall some notions for multi-valued mappings. Let $F: E \rightarrow 2^{Y}$ be a multi-valued mapping, where $2^{Y}$ denotes the collection of all subsets of $Y$. (i) $F$ is said to be upper semicontinuous at $x \in E$, if and only if, for each open set $U$ with $U \supset F(x)$, there exists an open neighborhood $O(x)$ of $x$ such that $U \supset F\left(x^{\prime}\right)$ for any $x^{\prime} \in O(x)$; (ii) $F$ is lower semicontinuous at $x \in E$, if and only if, for each open set $U$ with $U \cap F(x) \neq \emptyset$, there exists an open neighborhood $O(x)$ of $x$ such that $U \cap F\left(x^{\prime}\right) \neq \emptyset$ for any $x^{\prime} \in O(x)$; (iii) $F$ is continuous at $x \in E$, if and only if it is both upper semicontinuous and lower semicontinuous at $x$; (iv) $F$ is almost lower semicontinuous at $x \in E$, if and only if there is at least one $y \in F(x)$ such that, for each open neighborhood $U(y)$ of $y$, there exists an open neighborhood $O(x)$ of $x$ such that $U(y) \cap F\left(x^{\prime}\right) \neq \emptyset$, for all $x^{\prime} \in O(x) ;(\mathrm{v}), F$ is said to be metric upper semicontinuous at $x \in E$, if for each $\varepsilon>0$, there exists an open neighborhood $O(x)$ of $x$ such that $F\left(x^{\prime}\right) \subset B_{\varepsilon}(F(x))$ for all $x^{\prime} \in O(x)$, where $B_{\varepsilon}(F(x))$ denotes the $\varepsilon$-neighborhood of $F(x)$; (vi) $F$ is said to be metric lower semicontinuous at $x$ if for each $\varepsilon>0$, there exists an open neighborhood $O(x)$ of $x$ such that $F(x) \subset B_{\varepsilon}\left(F\left(x^{\prime}\right)\right)$ for all $x^{\prime} \in O(x)$.

Generally, if $F$ is upper semicontinuous (metric lower semicontinuous) at $x, F$ is metric upper semicontinuous (lower semicontinuous) at $x$.

Let $X$ be a nonempty, compact, and convex subset of the metric linear space $(E, d)$. Let $S: X \rightarrow C K(X)$ be a multivalued mapping which admits a continuous selection, where $C K(X)$ denotes the family of nonempty closed subsets of $X$. Denote by $M$ all such multi-valued mappings $S$ on $X$, clearly, $M$ includes Michael type fixed point mappings. Then for each $S \in M$, by the Brouwer's fixed point theorem, there is a fixed point $x^{*}$ of $S$; that is, $x^{*} \in S\left(x^{*}\right)$.

For each $S \in M$, let $F(S)=\{x \in X: x \in S(x)\}$. Then, $F$ defines a multi-valued mapping from $M$ to $X$. Clearly, for each $S \in M$, we have $F(S) \neq \emptyset$.

The following example shows that the fixed point set $F(S)$ for each $S \in M$ is not necessarily closed and not compact. In fact, the lack of compactness of $F(S)$ may result in some difficulties in the study of stability of these fixed points.

Example 1. Let $X=[0,1] \subset \mathbb{R}$ and $S \in M$ such that

$$
S(x)= \begin{cases}{[0,1],} & x \neq \frac{1}{2} \\ 0, & x=\frac{1}{2}\end{cases}
$$

We can check that $S$ is lower semicontinuous and has nonempty closed convex values, then, $S$ has a continuous section, to be prise, $f(x) \equiv 0, \forall x \in X$, hence, $S \in M$. Clearly, we have $F(S)=[0,1 / 2) \cup(1 / 2,1]$, which is open in $X$, hence, $F(S)$ is not compact.

We define the metric between any $S_{1}$ and $S_{2}$ in $M$ as

$$
\rho\left(S_{1}, S_{2}\right)=\sup _{x \in X} h\left(S_{1}(x), S_{2}(x)\right),
$$

where $h(A, B)$ is the Hausdorff metric induced by $d$. Then $(M, \rho)$ is a metric space. The following two examples reveal that the fixed points mapping $F$ lacks both upper and lower semicontinuities.

Example 2. Let $X=[0,1] \subset \mathbb{R}$. Let $S \in M$ satisfying $S(x)=$ $[0, x], \forall x \in X$. Then, $F(S)=X$. Define $S_{n} \in M, n=1,2, \ldots$, such that

$$
S_{n}(x)= \begin{cases}S(x), & x \in\left[0, \frac{1}{2}\right), \\ {\left[0, x-\frac{1}{n+1}\right],} & x \in\left[\frac{1}{2}, 1\right] .\end{cases}
$$

Clearly, $S_{n} \in M, n=1,2, \ldots$ Then, $F\left(S_{n}\right)=[0,(1 / 2))$, $n=1,2, \ldots$, and we have $S_{n} \rightarrow S$ as $n$ gets close to infinity. Let $V_{\varepsilon}=(1-\varepsilon, 1]$, then $V_{\varepsilon}$ is open in $X$ and $F(S) \cap V_{\varepsilon}=V_{\varepsilon} \neq \emptyset$, where $\varepsilon>0$. However, if $\varepsilon$ is small enough, we will obtain that $F\left(S_{n}\right) \cap V_{\varepsilon}=\emptyset, n=1,2, \ldots$. Thus, $F$ is not lower semicontinuous at $S$.

Remark 3. For the $S \in M$ in Example 2, for each fixed point $x \in F(S)$ and small enough $\varepsilon$ neighborhood $B_{\varepsilon}(x)$ of $x$, we have $B_{\varepsilon}(x) \cap F(S) \neq \emptyset$, similarly, we can construct $S_{n} \in M$ such that $F\left(S_{n}\right) \cap B_{\varepsilon}(x)=\emptyset, n=1,2, \ldots$ That is, $F$ is not also almost lower semicontinuous at $S$.

Example 4. Let $X=[0,1] \subset \mathbb{R}$. Let $S \in M$ such that

$$
S(x)= \begin{cases}0, & x \in\left[0, \frac{1}{2}\right], \\ {[0,1],} & x \in\left(\frac{1}{2}, 1\right] .\end{cases}
$$

Then, $F(S)=\{0\} \cup(1 / 2,1]$. For each $n=2,3, \ldots$, define a multi-valued mapping $S_{n}$ on $X$ as

$$
S_{n}(x)= \begin{cases}0, & x \in\left[0, \frac{1}{2}-\frac{1}{n}\right], \\ {\left[0, \frac{1}{n}\right],} & x=\frac{1}{2}, \\ {[0,1],} & x \in\left(\frac{1}{2}-\frac{1}{n}, \frac{1}{2}\right) \cup\left(\frac{1}{2}, 1\right] .\end{cases}
$$

Clearly, we can check that $S_{n} \in M, n=2,3, \ldots$ and $\rho\left(S_{n}, S\right) \rightarrow$ $0(n \rightarrow \infty)$. Take an open set $V=[0,1 / 5) \cup(1 / 2,1]$, then $F(S) \subset V$. However, $F\left(S_{n}\right)=\{0\} \cup(1 / 2-1 / n, 1 / 2) \cup(1 / 2,1] \not \subset$ $V, n=4,5, \ldots$. Therefore, $F$ is not upper semicontinuous at $S$.

Noting that $F$ is neither upper semicontinuous nor lower semicontinuous on $M$, in order to study the continuity of $F$ further, for each $S \in M$, we denote by $G(S)$ the set of all continuous selections of $S$. That is, $G$ defines a multivalued mapping from $M$ to $C(X, X)$, where $C(X, X)$ is the 
usual space of continuous functions from $X$ to $X$, on which the uniform metric is adopted. Clearly, $G(S) \neq \emptyset$ for each $S \in$ $M$. Define a multi-valued mapping $T: C(X, X) \rightarrow 2^{X}$ such that $T(f)$ denotes the set of fixed points of $f \in C(X, X)$ on $X$. Obviously, $T(f)$ is nonempty and compact for each $f \in C(X, X)$.

Lemma 5 (see [7]). Let $P$ be a complete metric space, $Y$ be a metric space, and $F: P \rightarrow 2^{Y}$ be metric upper semicontinuous. Then, there exists a dense residual set $Q \subset P$ such that $F$ is metric lower semicontinuous at each $x \in Q$.

\section{Stability of Fixed Points for multi-valued Mappings}

Theorem 6. The graph of $G, \operatorname{gr}(G)=\{(S, f) \in M \times C(X, X)$ : $f \in G(S)\}$, is closed.

Proof. Let $\left(S_{n}, f_{n}\right) \in \operatorname{gr}(G), n=1,2, \ldots$, with $\left(S_{n}, f_{n}\right) \rightarrow$ $\left(S_{0}, f_{0}\right) \in M \times C(X, X)$. Then, $f_{n}$ is continuous on $X$, and $f_{n}(x) \in S_{n}(x)$ for each $x \in X$ and $n=1,2, \ldots$. We need to show that $f_{0} \in G\left(S_{0}\right)$. For each $x \in X$, we can obtain that

$$
\begin{aligned}
d\left(f_{0}(x), S_{0}(x)\right) \leq & d\left(f_{0}(x), f_{n}(x)\right) \\
& +d\left(f_{n}(x), S_{n}(x)\right)+h\left(S_{n}(x), S_{0}(x)\right)
\end{aligned}
$$

where $d(z, A)=\inf _{a \in A}\{d(z, a)\}$. As $n$ get close to infinity, we have $d\left(f_{0}(x), S_{0}(x)\right) \rightarrow 0$ because $\left(S_{n}, f_{n}\right) \rightarrow\left(S_{0}, f_{0}\right)$ and $f_{n}(x) \in S_{n}(x)$. Since $S_{0}(x)$ is closed, we have $f_{0}(x) \in S_{0}(x)$. Additionally, $f_{0}$ is also continuous, which follows from $f_{n} \rightarrow$ $f_{0}$. Therefore, $f_{0}$ is a continuous selection of $S_{0}$. The proof is completed.

Theorem 7. The graph of $T, \operatorname{gr}(T)=\{(f, x) \in C(X, X) \times X$ : $x \in T(f)\}$, is closed.

Proof. For $\left(f_{n}, x_{n}\right) \in \operatorname{gr}(T), n=1,2, \ldots$, with $\left(f_{n}, x_{n}\right) \rightarrow$ $\left(f_{0}, x_{0}\right) \in C(X, X) \times X$, clearly, it holds that $f_{n}\left(x_{n}\right)=x_{n}$. Noting the following inequality:

$$
\begin{aligned}
& d\left(x_{0}, f_{0}\left(x_{0}\right)\right) \\
& \quad \leq d\left(x_{0}, x_{n}\right)+d\left(x_{n}, f_{n}\left(x_{n}\right)\right) \\
& \quad+d\left(f_{n}\left(x_{n}\right), f_{0}\left(x_{n}\right)\right)+d\left(f_{0}\left(x_{n}\right), f_{0}\left(x_{0}\right)\right),
\end{aligned}
$$

and since $\left(f_{n}, x_{n}\right) \rightarrow\left(f_{0}, x_{0}\right)$ with the continuity of $f_{0}$, we have $d\left(x_{0}, f_{0}\left(x_{0}\right)\right) \rightarrow 0$. Then, $f_{0}\left(x_{0}\right)=x_{0}$. Therefore, $\left(f_{0}, x_{0}\right) \in \operatorname{gr}(T)$.

Remark 8. For the multi-valued mapping $S$ in Example 4, we can check that $T \circ G(S)=\cup_{f \in G(S)} T(f)=\{0\} \cup(1 / 2,1]$. For each multi-valued mapping $S_{n}$ with $n>3$ in Example 4, we have $T \circ G\left(S_{n}\right)=\{0\} \cup(1 / 2-1 / n, 1 / 2) \cup(1 / 2,1]$. Therefore, $T \circ G$ is not upper semicontinuous on $M$, though the graphs of $T$ and $G$ are closed.
For each $S \in M$, though $S$ admits a continuous selection, there is no semicontinuities for fixed point mappings from examples in Preliminaries, we consider a kind of space $M^{\prime}$ for which each $S \in M^{\prime}$ admits a Lipschitz selection. That is,

$$
M^{\prime}=\{S: X \longrightarrow C K(X): S \text { has a } K \text {-Lipschitz selection }\} \text {, }
$$

where a $K$-Lipschitz selection $f$ of $S$ means that $f(x) \in S(x)$, $\forall x \in X$ such that there exists a $k$ with $k \leq K$ satisfying that $d(f(x), f(y))<k d(x, y)(k \geq 0)$ for any $x, y \in X$. Obviously, each $K$-Lipschitz selection $f$ of $S$ is a continuous selection.

For the space $M^{\prime}$, we can also use $\rho\left(S_{1}, S_{2}\right)$ to measure the metric between any two $S_{1}, S_{2} \in M^{\prime}$. Then $\left(M^{\prime}, \rho\right)$ is also a metric space. For each $S \in M^{\prime}$, let $F^{\prime}(S)$ denote the set of fixed points, obviously, $F^{\prime}(S)$ is also not necessarily compact; denote by $G^{\prime}(S)$ the set of $K$-Lipschitz selections of $S$; that is, $G^{\prime}$ defines a multi-valued mapping from $M$ to $C(X, X)$. By the construction of $M^{\prime}$, we know that $T \circ G^{\prime}(S)=\cup_{f \in G^{\prime}(S)} T(f) \neq \emptyset$ for each $S \in M^{\prime}$.

Definition 9. For each $S \in M^{\prime}$, a set $e(S)$ is called an essential fixed point set of $S$ with respect to $M^{\prime}$ if and only if it satisfies the following conditions.

(1) $e(S)$ is nonempty closed subset of $F^{\prime}(S)$;

(2) For any open set $U$ with $U \supset e(S)$, there exists an open neighborhood $O(S)$ in $M^{\prime}$ such that $U \cap F^{\prime}\left(S^{\prime}\right) \neq \emptyset$, for any $S^{\prime} \in O(S)$.

If $e(S)$ is a singleton set $\left\{x^{*}\right\}, x^{*}$ is called an essential fixed point of $S$ with respect to $M^{\prime}$. An essential fixed point set $e^{*}(S)$ is said to be minimal if it is a minimal element in the family of essential sets of $S$ ordered by set inclusion in $F^{\prime}(S)$.

Remark 10. An essential fixed point $x^{*}$ of $S$ means that for each multi-valued mapping near $S$ there is a fixed point near the point $x^{*}$. Let $A, B \subset F^{\prime}(S)$ be two nonempty sets with $A \subset B$, if $A$ is essential, then $B$ follows.

Theorem 11. The metric space $\left(M^{\prime}, \rho\right)$ is complete.

Proof. Let $\left\{S_{n}\right\}_{n=1}^{\infty}$ be a Cauchy sequence in $M^{\prime}$. Then, for any $\varepsilon>0$, there exists a number $N$ such that $\rho\left(S_{n}, S_{m}\right)<\varepsilon$ for any $n, m>N$. That is,

$$
h\left(S_{n}(x), S_{m}(x)\right)<\varepsilon, \quad \forall x \in X .
$$

Therefore, for each $x \in X,\left\{S_{n}(x)\right\}_{n=1}^{\infty}$ is a Cauchy sequences in $K(X)$, where $K(X)$ is the collection of nonempty and compact subsets in $X$. Clearly, $K(X)$ is complete. Then for each $x \in$ $X$, there is a $S_{0}(x) \in K(X)$ such that $h\left(S_{n}(x), S_{0}(x)\right) \rightarrow 0$. That is, there exists a mapping $S_{0}: X \rightarrow K(X)$ such that $\rho\left(S_{n}, S_{0}\right) \rightarrow 0$.

For each $n=1,2, \ldots$, let $f_{n}$ be a $K$-Lipschitz selection of $S_{n}$. Since the collection of Lipschitz functions on $X$ bounded with $K$ is compact, there is a convergent subsequence $\left\{f_{n_{k}}\right\}_{k=1}^{\infty}$ of $\left\{f_{n}\right\}_{n=1}^{\infty}$ with $f_{n_{k}} \rightarrow f_{0} \in C(X, X)$. Noting $\left(S_{n_{k}}, f_{n_{k}}\right) \rightarrow$ $\left(S_{0}, f_{0}\right)$, similar to the proof of Theorem 6 , we can prove that 
$f_{0}(x) \in S_{0}(x), \forall x \in X$. Additionally, for any two points $x_{1}, x_{2} \in X$, since

$$
\begin{aligned}
& d\left(f_{0}\left(x_{1}\right), f_{0}\left(x_{2}\right)\right) \\
& \leq d\left(f_{0}\left(x_{1}\right), f_{n_{k}}\left(x_{1}\right)\right) \\
& \quad+d\left(f_{n_{k}}\left(x_{1}\right), f_{n_{k}}\left(x_{2}\right)\right) \\
& \quad+d\left(f_{n_{k}}\left(x_{2}\right), f_{0}\left(x_{2}\right)\right) \\
& \leq d\left(f_{0}\left(x_{1}\right), f_{n_{k}}\left(x_{1}\right)\right)+K d\left(x_{1}, x_{2}\right) \\
& \quad+d\left(f_{n_{k}}\left(x_{2}\right), f_{0}\left(x_{2}\right)\right),
\end{aligned}
$$

and $f_{n_{k}} \rightarrow f_{0}$, we have $d\left(f_{0}\left(x_{1}\right), f_{0}\left(x_{2}\right)\right) \leq K d\left(x_{1}, x_{2}\right)$. That is, $f_{0}$ is a $K$-Lipschitz selection of $S_{0}$. Then, $S_{0} \in M^{\prime}$. The space $\left(M^{\prime}, \rho\right)$ is complete.

Theorem 12. The multi-valued mapping $T \circ G^{\prime}: M^{\prime} \rightarrow 2^{X}$ is upper semicontinuous with compact values.

Proof. Following the proof of Theorems 6 and 7, both $T$ and $G^{\prime}$ are closed. Noting that $X$ is compact and the collection of Lipschitz functions bounded with $K$ on $X$ is also compact, we have $T$ and $G^{\prime}$ upper semicontinuous with compact values. It follows that $T \circ G^{\prime}$ is upper semicontinuous and has compact values on $M^{\prime}$.

By Theorem 12, in fact we find a special submapping of the fixed point mapping $F^{\prime}$ on $M^{\prime}\left(T \circ G^{\prime}(S) \subset F^{\prime}(S), \forall S \in M^{\prime}\right)$. A result can be obtained as follows.

Theorem 13. For almost all $S \in M^{\prime}$, there exists at least one essential fixed point of $S$ with respect to $M^{\prime}$.

Proof. Noting that each upper semicontinuous mapping on $X$ with compact values is also metric upper semicontinuous, by Lemma 5, Theorems 11 and 12, there exists a dense residual set $Q$ in $M^{\prime}$ such that $T \circ G^{\prime}$ is metric lower semicontinuous on it; definitely, we have $T \circ G^{\prime}$ as also lower semicontinuous on $Q \subset M^{\prime}$. Then for each $S \in Q, x \in T \circ G^{\prime}(S)$ and any open neighborhood $O(x)$ of $x$ in $X$, there is an open $V \supset S$ such that $T \circ G^{\prime}\left(S^{\prime}\right) \cap O(x) \neq \emptyset$ for all $S^{\prime} \in V$. Noting that $T \circ G^{\prime}\left(S^{\prime}\right) \subset$ $F^{\prime}\left(S^{\prime}\right)$, we have $F^{\prime}\left(S^{\prime}\right) \cap O(x) \neq \emptyset, \forall S^{\prime} \in V$. Then, $x$ is definitely an essential fixed point of $S$ with respect to $M^{\prime}$.

Theorem 14. For each $S \in M^{\prime}$, there exists at least one minimal essential set $m(S)$ of $F^{\prime}(S)$ with respect to $M^{\prime}$.

Proof. From Theorem 12, for each $S \in M, T \circ G^{\prime}$ is upper semicontinuous with compact values. Then, the set $T \circ G^{\prime}(S)$ is closed. Hence, $T \circ G^{\prime}(S)$ is essential with respect to $M^{\prime}$ from Definition 9. For each essential set $e(S) \subset F^{\prime}(S)(e(S) \neq T$ 。 $\left.G^{\prime}(S)\right)$, if $e(S) \not \subset T \circ G^{\prime}(S)$, then $T \circ G^{\prime}(S)$ is a minimal essential set of $F^{\prime}(S)$.

If $T \circ G^{\prime}(S)$ is not a minimal essential set of $F^{\prime}(S)$. Let $\Gamma$ denote the collection of all essential subsets in $T \circ G^{\prime}(S)$. Then each decreasing chain of elements in $\Gamma$ has a lower bound, which is the intersection of the chain and is compact.
Therefore, there is a minimal element $m(S) \subset T \circ G^{\prime}(S)$ in $\Gamma$ by Zorn's lemma. For each essential set $e(S)$ in $F^{\prime}(S)$ with $e(S) \neq m(S)$, if $e(S) \subset T \circ G^{\prime}(S)$, we can obtain that $e(S) \not \subset m(S)$ since $m(S)$ is minimal; clearly, if $e(S) \not \subset T \circ G^{\prime}(S)$, we also have $e(S) \not \subset m(S)$. Then, the set $m(S)$ is minimally essential. Therefore, $T \circ G^{\prime}(S)$ itself is either a minimally essential set or a set which includes at least one.

Remark 15. (a) By Theorem 13, a result for generic stabilities for fixed points of mappings on $M^{\prime}$ is obtained. (b) For the multi-valued mapping $S \in M$ in Example 4, clearly, $f(x) \equiv 0$, $x \in X$ is a $K$-Lipschitz selection of $S(K \geq 0)$, hence, $S \in M^{\prime}$ and $F^{\prime}(S)=\{0\} \cup((1 / 2), 1]$, a noncompact set.

Remark 16. For the space of upper semicontinuous multivalued mapping with closed convex values on $X$ (see [13]), we know that the upper semicontinuity of the fixed point mapping results in the compactness of fixed point set and the existence of essential sets of fixed points. However, for the space $M$ (including Michael type mappings, lower semicontinuous multi-valued mappings with closed convex values), the fixed point mapping is neither upper semicontinuous nor lower semicontinuous. In the space $M^{\prime}$, Theorem 14 shows the existence of essential sets of $F^{\prime}(S)$, and each closed set including a minimal essential set of $F^{\prime}(S)$ is essentially stable.

Finally, as an application, we consider a kind of differential inclusion as follows:

$$
\begin{gathered}
\dot{x}(t) \in S(x(t)), \\
x(0)=x_{0},
\end{gathered}
$$

where the values of $x(t), \forall t \in[0, a]$ is defined in a closed region $X \subset R^{n}$, and $S: X \rightarrow C K(X)$ is a multi-valued mapping. Let $F_{o}(S)$ denote the solution set of this differential inclusion for each $S \in M^{\prime}$. Then, if $f$ is a $K$-Lipschitz selection of $S$, each initial value problem,

$$
\begin{gathered}
\dot{x}(t)=f(x(t)), \\
x(0)=x_{0},
\end{gathered}
$$

must have a unique solution on $t \in[0, a], 0<a<\infty$. Noting this fact, for each $S \in M^{\prime}$, if we also use $G^{\prime}(S)$ to denote all $K$-Lipschitz selections of $S$, then we now denote $T \circ G^{\prime}(S)$ the solution set of the above differential inclusion with respect to all $f \in G^{\prime}(S)$. Then, we have $T \circ G^{\prime}(S) \subset F_{o}(S), \forall S \in M^{\prime}$. If fixed points is instead by solutions in Definition 9 , we can define essential solution sets $e(S)$, minimal essential solution sets $e^{*}(S)$, and essential solutions of above differential inclusion in $F_{o}(S)$ with respect to $M^{\prime}$.

In the space $\left(M^{\prime}, \rho\right)$, by Theorems 13 and 14 and the meaning of essentially stabilities, we can obtain the following result.

Theorem 17. For almost all $S \in M^{\prime}$, there has been at least an essential solution in $F_{o}(S)$; for each $S \in M^{\prime}$, there exists a minimal essentially stable set $e^{*}(S) \subset F_{o}(S)$ which can resist the perturbation of $S$ in $M^{\prime}$. 


\section{Conflict of Interests}

The author declares that there is no conflict of interests regarding the publication of this paper.

\section{Acknowledgments}

This project is supported by the Natural Science Foundation of Guangxi (no. 2012GXNSFBA053013 and no. 2013GXNSFBA19004) and Doctoral Research Fund of Guilin University of Technology.

\section{References}

[1] M. K. Fort, Jr., "Essential and non essential fixed points," The American Journal of Mathematics, vol. 72, pp. 315-322, 1950.

[2] G. Isac and G. X. Z. Yuan, "The essential components of coincident points for weakly inward and outward set-valued mappings," Applied Mathematics Letters, vol. 12, no. 4, pp. 121126, 1999.

[3] Z. Lin, H. Yang, and J. Yu, "On existence and essential components of the solution set for the system of vector quasiequilibrium problems," Nonlinear Analysis, Theory, Methods and Applications, vol. 63, no. 5-7, pp. e2445-e2452, 2005.

[4] O. Carbonell-Nicolau, "Essential equilibria in normal-form games," Journal of Economic Theory, vol. 145, no. 1, pp. 421-431, 2010.

[5] M. K. Fort, Jr., "Points of continuity of semi-continuous functions," Publicationes Mathematicae Debrecen, vol. 2, pp. 100-102, 1951.

[6] E. Maskin and J. Tirole, "Markov perfect equilibrium. I. Observable actions," Journal of Economic Theory, vol.100, no. 2, pp. 191219, 2001.

[7] S. W. Xiang, W. S. Jia, J. H. He, S. Y. Xia, and Z. Y. Chen, "Some results concerning the generic continuity of set-valued mappings," Nonlinear Analysis. Theory, Methods and Applications, vol. 75, no. 8, pp. 3591-3597, 2012.

[8] P. Q. Khanh and N. H. Quan, "Generic stability and essential components of generalized KKM points and applications," Journal of Optimization Theory and Applications, vol. 148, no. 3, pp. 488-504, 2011.

[9] Q. Q. Song and L. S. Wang, "On the stability of the solution for multiobjective generalized games with the payoffs perturbed," Nonlinear Analysis. Theory, Methods and Applications, vol. 73, no. 8, pp. 2680-2685, 2010.

[10] G. Isac and X. Z. Yuan, "The generic stability of fixed points for upper semicontinuous mappings in hyperconvex metric spaces," Numerical Functional Analysis and Optimization, vol. 21, no. 7-8, pp. 859-868, 2000.

[11] S. Kinoshita, "On essential components of the set of fixed points," Osaka Mathematical Journal, vol. 4, pp. 19-22, 1952.

[12] B. O'Neill, "Essential sets and fixed points," The American Journal of Mathematics, vol. 75, pp. 497-509, 1953.

[13] J. H. Jiang, "Essential component of the set of fixed points of the multivalued mappings and its application to the theory of games," Scientia Sinica, vol. 12, pp. 951-964, 1963.

[14] S. Govindan and R. Wilson, "Essential equilibria," Proceedings of the National Academy of Sciences of the United States of America, vol. 102, no. 43, pp. 15706-15711, 2005.
[15] E. Michael, "Selected Selection Theorems," The American Mathematical Monthly, vol. 63, no. 4, pp. 233-238, 1956.

[16] E. Michael, "Continuous selections I," Annals of Mathematics, vol. 63, pp. 361-382, 1956. 


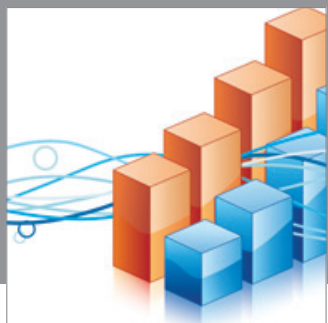

Advances in

Operations Research

mansans

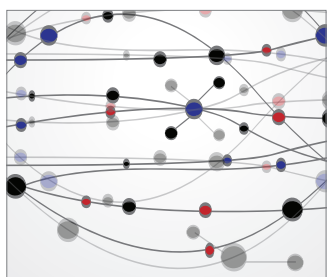

The Scientific World Journal
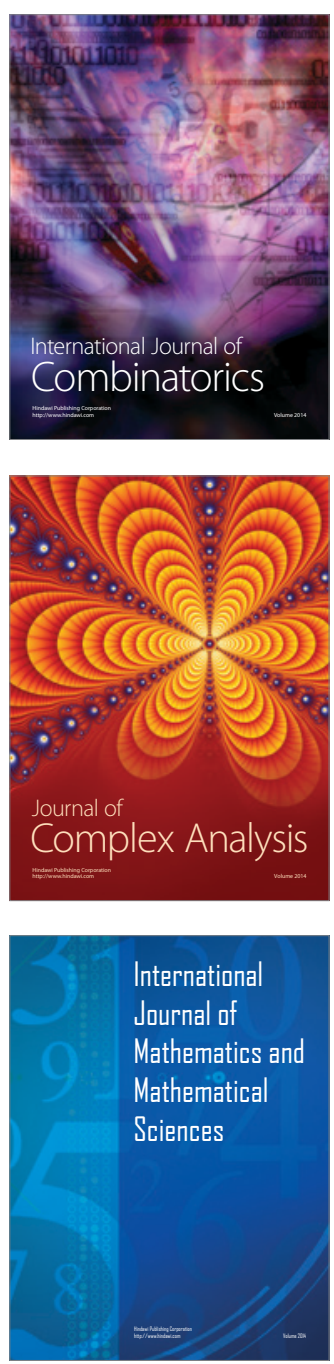
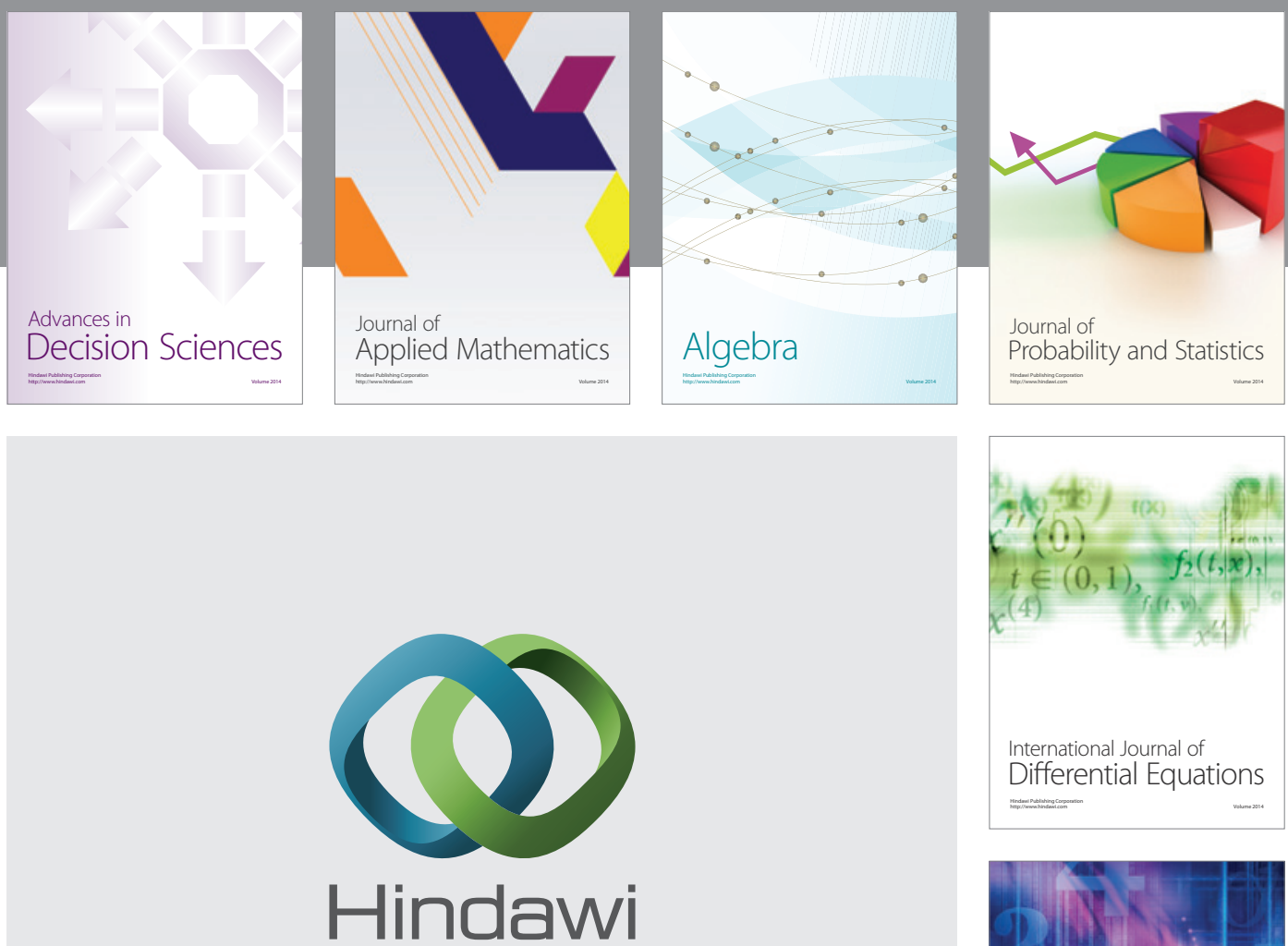

Submit your manuscripts at http://www.hindawi.com
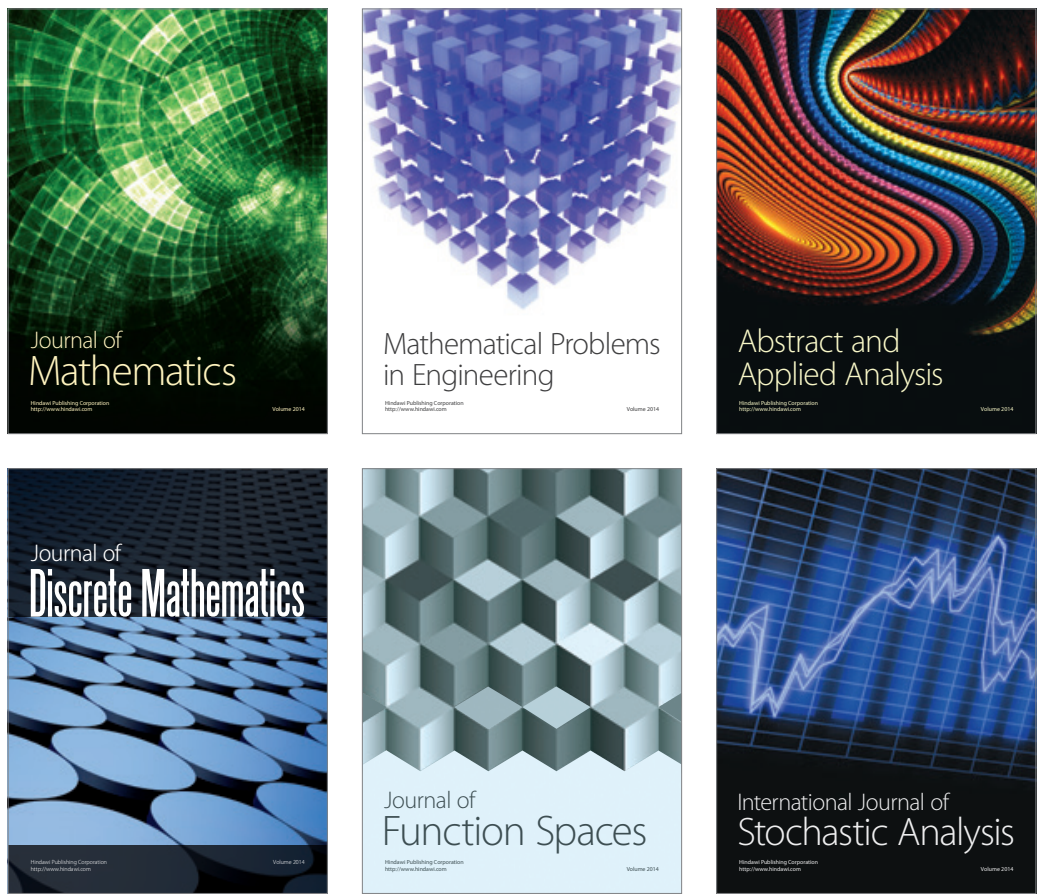

Journal of

Function Spaces

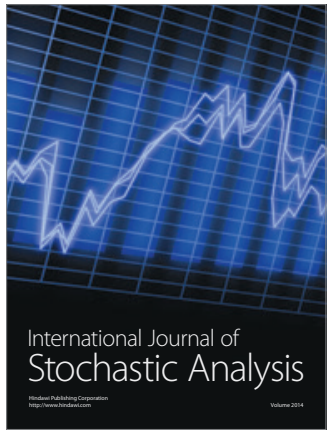

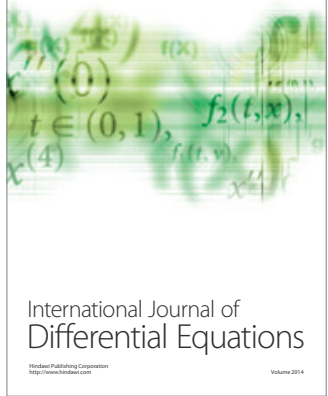
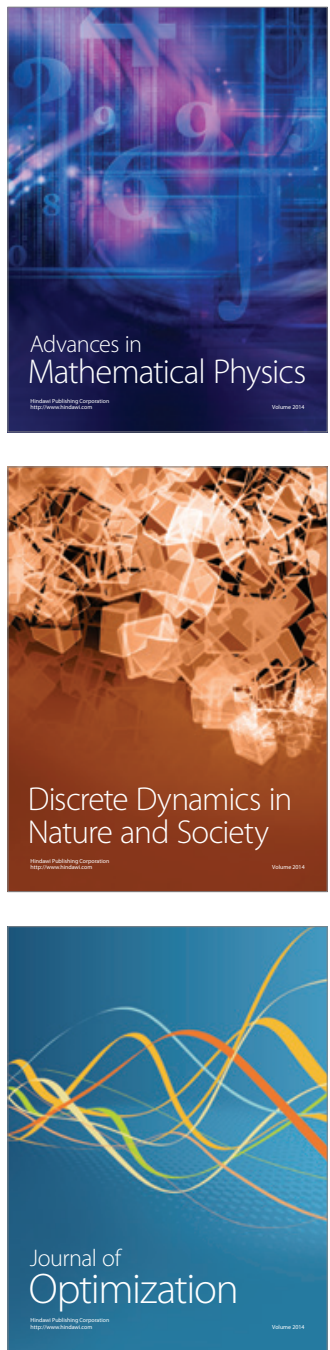